\title{
Shear Bond Strength of different resin-based materials Processed on Poly-ether-ether ketone frameworks (IN VITRO STUDY)
}

\author{
Mohamed A. Eltombakshy $B S c^{1^{*}}$, Mohamed S. Elattar $P h D^{2}$, Dawlat M. Ahmed $P h D^{3}$
}

\begin{abstract}
INTRODUCTION: Removable partial dentures (RPDs) are fabricated to replace the lost teeth of a partially edentulous individual. The serviceability of an RPD is dependent on the bond strength at the resin-framework interface. The esthetically unacceptable display of metal clasps, the increased weight of the prosthesis, the potential for metallic taste, and allergic reactions to metals led to the introduction of a number of thermoplastic materials in clinical practice such as nylon and acetal resins. An alternative restoration material (poly-ether-etherketone [PEEK]) has been successfully used over the last years.

OBJECTIVES: to evaluate the shear bond strength and the effect of aging on bond strength of two different resin based materials to polyether-ether-ketone [PEEK].

MATERIALS AND METHODS: Fifty two PEEK cylinders was prepared. Specimens was randomly assigned into two parallel groups, 26 specimens each according to the resin materials used. The bond strength and aging of resin material to poly-ether-ether-ketone [PEEK] was evaluated.

RESULTS: the initial shear bond strength of PMMA to PEEK was significantly higher than that of composite resin to PEEK. However, after aging process the shear bond strength of PMMA to PEEK decreased while that of composite resin to PEEK remained unchanged. There was no significant difference between the shear bond strength of both PMMA and composite resin to PEEK after aging.

CONCLUSIONS: PMMA can be used as a veneering material with PEEK with comparable results to traditionally used composite resin. Moreover, owing to the adequate bond strength between PEEK and PMMA, PEEK frameworks could be relined in the traditional method similar to metal frameworks
\end{abstract}

KEYWORDS: PEEK, PMMA, Composite resin, Shear bond strength, Thermocycling, in vitro.

1. Postgrad. Candidate, Faculty of Dentistry, Alexandria University

2. Professor of Prosthetic Dentistry, Faculty of Dentistry Alexandria University

3. Lecturer of Dental Biomaterials, Faculty of Dentistry, Alexandria University

Corresponding author:

E-mail: m.eltombakshy@gmail.com

\section{INTRODUCTION}

Poly-ether-ether-ketone (PEEK) is a polymer from the group poly-aryl-ether-ketone (PAEK). It is a relatively new family of high-temperature thermoplastic polymers, consisting of an aromatic backbone molecular chain, interconnected by ketone and ether functional groups (1).

Historically, the availability of PEEK arrived at a time when there was growing interest in the development of "isoelastic" hip stems and fracture fixation plates with stiffness comparable with bone (2).

By the late 1990s, PEEK had emerged as the leading high-performance thermoplastic candidate for replacing metal implant components, especially in orthopedics (3) and trauma (4). Not only was the material resistant to simulated in vivo degradation, including damage caused by lipid exposure, but starting in April 1998, PEEK was offered commercially as a biomaterial for implants (Invibio Ltd., Thornton Cleveleys, United Kingdom) (5). Facilitated by a stable supply, research on PEEK biomaterials flourished and is expected to continue to advance in the future (6).

The monomer unit of ether-ether-ketone monomer (Figure 1) polymerizes via step-growth dialkylation reaction of bis-phenolates to form PEEK. A common synthesis route for PEEK is the reaction between 4,40difluorobenzophenone and the disodium salt of hydroquinone in a polar solvent at $300{ }^{\circ} \mathrm{C}$. It is a semicrystalline material having a melting point around $335{ }^{\circ} \mathrm{C}$. PEEK can modified either by the addition of functionalized monomers (pre-polymerization) or post-polymerization modifications by chemical processes such as sulfonation, amination and nitration (7).

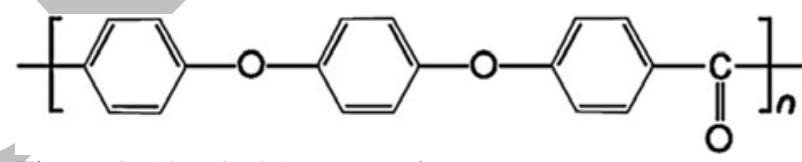

Figure 1: Chemical Structure of PEEK

Due to its elastic modulus, similar to that of cortical bone, it plays an important role as a viable alternative to conventional implant materials such as titanium in the field of orthopedics (8) and traumatology (9). Also in the field of dentistry, where traditionally a wide range of alloplastic materials is used, the application of PEEK increases replacing those conventional dental materials (10). This can be associated with distinct problems. Besides hypersensitivities against distinct components of dental composites on the one hand, even titanium, which is known as a proven biocompatible metal, has recently been suspected to provoke inflammatory reactions on the other hand (11). Additionally, more and more patients desire metal free reconstructions (12), to avoid the risk of oral galvanism for instance (13). Due to its mechanical properties, which can be influenced by adding different compound materials (1), PEEK might represent a viable biomaterial, not only able to replace conventional polymers, but also even metals, alloys and ceramics in the field of dentistry. 
The use of RPDs in clinical practice remains a viable and predictable treatment modality. Data on future needs for RPDs indicate that the need for RPDs is actually predicted to increase as the geriatric population increases which includes a high percentage of partially edentulous patients (14). The use of alternatives to RPD such as implants may not always be practical for a number of reasons and the benefits of RPDs are well documented (15).

Traditional RPD with Co-Cr frameworks and clasps have been an inexpensive and predictable treatment option for the rehabilitation of partially edentulous patients (16). The esthetically unacceptable display of metal clasps, increased weight of the prosthesis, potential for metallic taste, and allergic reactions to metals led to the introduction of a number of thermoplastic materials in clinical practice such as nylon and acetal resins. Nylons provide improved esthetics and reduction of rotational forces on the abutment teeth due to their low elastic modulus. The major disadvantage of a nylon RPD is the inability for a reline procedure and the lack of occlusal rests as well as rigid frameworks that could lead to occlusal instability and sinking, especially in Kennedy class I and II cases. On the other hand, acetal resins present adequate mechanical strength to form a framework more rigid than nylon with retentive clasps, connectors, and supportive elements; however, the acetal resin material lacks natural translucency and vitality (17).

Alternatively, RPDs can be constructed by using PEEK. Its elasticity can reduce stresses transferred to the abutment teeth. Furthermore, the white color of PEEK frameworks provides a different esthetic approach than the conventional metal framework display does. Additional advantages of this polymer material are elimination of allergic reactions and metallic taste, high polishing qualities, low plaque affinity, and good wear resistance (18).

RPDs can be constructed more efficiently by using PEEK computer-aided design and computer-aided manufacture systems (1). The use of CAD-CAM as a manufacturing process could lead to further process simplification, providing a cost and time-saving approach and eliminating tool wear (19).

PEEK features a white colored appearance as compared to metal reconstructions. However, from the aesthetic point of view, it still requires veneering owing to its low translucency and grayish pigmentation. Consequently, durable bonding must be achieved to ensure an adequate functional outcome and long-term stability. The latter can be established by chemical adhesion, (micro) mechanical retention, or a combination of both and depends on the composition and interaction of the materials used (20).

In this context, one disadvantage of using PEEK in prosthetic dentistry is a difficulty to achieve adequate bond strength to resin materials owing to its low surface energy and resistance to surface modification by different chemical treatments (21).

In view of the limited data available on bonding to PEEK materials and the lack of data on bonding between PEEK and acrylic resin, the purpose of this study is to evaluate the bond strength and the effect of aging on bond strength of two different resin based materials (composite resin and acrylic resin) to PEEK.

\section{MATERIALS AND METHODS \\ Materials}

Materials which were used in this study are:

1. Framework material:

- BioHPP; modified PEEK (BioHPP; Bredent GmbH, Senden, Germany)

2. PMMA and composite primer for bonding (Visio.link; Bredent GmbH, Senden, Germany)

3. Resin materials:

a.Heat cure acrylic material (Acrostone; Acrostone Dental Manufacturer, Egypt Acrostone; Acrostone Dental Manufacturer, Egypt)

b. Composite resin (Visio.lign; Bredent $\mathrm{GmbH}$, Senden, Germany)

\section{Equipments}

- Universal testing machine (AG1, Shimadzu Corporation, Kyoto, Kyoto Prefecture, Japan) to test shear bond strength

- Thermocycling machine (Custom made; Dental Biomaterials Department, Faculty of Dentistry, Alexandria University, Egypt) for aging process

\section{Methods}

\section{Preparation of the framework specimens} Modified PEEK specimens

Fifty two patterns were fabricated as cylinders $(10 \mathrm{~mm}$ diameter and $10 \mathrm{~mm}$ height) by using hard inlay wax (Pyrax Inlay wax, Pyrax Polymars, Uttarakhand, India). The inlay wax was melted in a hot water bath and poured into a custom-made mould with the required dimensions. Then the wax patterns were invested in a specially designed dental flask and sprues were made to allow the flow of the material into the required spaces. After complete burning out of the wax, the modified PEEK material containing 20\% ceramic fillers (BioHPP; Bredent GmbH, Senden, Germany) was pressed into the flask by melting and vacuum pressing. The overall burnout schedule before pressing following the manufacturer's instructions. Each cylinder was then cleaned ultrasonically in distilled water for $60 \mathrm{~s}$, and then air dried. Standardization of bonding area

In order to standardize the bond area for the specimens, a Double-sided tape with a 6-mm diameter hole was attached to one flat surface of each cylinder to create fifty two specimens with a defined bond area $(19.6 \mathrm{~mm} 2)$. For this purpose, a Teflon O-ring (5.0-mm internal diameter, $1 \mathrm{~mm}$ thick) was positioned on these flat surfaces in such a way that its hole was contiguous with the hole in the doublesided tape.

\section{Grouping}

The specimens were divided into two groups; 26 specimens each:

- Group I (Control group); modified PEEK specimens bonded to composite resin (Visio.lign)

- Group II (Study Group); modified PEEK specimens bonded to heat cured PMMA.

After the specimens preparation, each group was further divided into 2 subgroups (13 specimens each), one subgroup was tested after initial bonding and the other subgroup was tested after thermocycling process

\section{Surface treatment}

The surface of all the specimens were then sandblasted by using $110 \mu$ aluminum oxide (Al2O3) at $0.25 \mathrm{MPa}$ for 30 seconds at a distance of $1 \mathrm{~cm}$, and then cleaned with water in an ultrasonic bath for 10 minutes.

Resin Bonding

PMMA 
PMMA (23 g/10 mL) (Acrostone; Acrostone Dental Manufacturer, Egypt) was packed and polymerized according to the manufacturer's instructions. Before packing, the bonding agent; Visio.Link (Bredent GmbH, Senden, Germany), was applied on the surface of the specimens according to manufacturer's instructions. Once polymerization was complete, the specimens were stored in distilled water at room temperature for 24 hours.

\section{Composite Resin}

Visio.lign (Bredent GmbH, Senden, Germany) was used according to the manufacturer's instructions. Before composite resin application, bonding agent; Visio.Link (Bredent GmbH, Senden, Germany), was applied on the surface of the specimens according to the manufacturer's instructions. Once polymerization was complete, the specimens were stored in distilled water at room temperature for 24 hours.

\section{Shear bond strength testing}

Shear Bond strength of randomly selected thirteen specimens of each group was determined by placing each specimen in a universal testing machine (AG1, Shimadzu Corporation, Kyoto, Kyoto Prefecture, Japan) at a crosshead speed of $1.0 \mathrm{~mm} / \mathrm{min}$ until the specimen failed.

The Shear bond strength is then determined by the equation:

$\tau=\mathrm{Pc} / \mathrm{A}$

Where Pc is the critical load to debonding and A is the bonded area.

\section{Aging and Shear bond strength testing}

Shear Bond strength of the other thirteen specimens of each group was determined after thermocycling in distilled water for 1000 cycles between $5^{\circ} \mathrm{C}$ and $55^{\circ} \mathrm{C}$. The specimens were then placed in a universal testing machine as previously mentioned

\section{Data management \& statistical analysis}

The data was processed, and analyzed using Statistical Package for Social Sciences program SPSS (15.0) software (SPSS Inc., Chicago IL, USA). The study included descriptive and analytical data. A P-value of less than 0.05 was considered statistically significant.

\section{RESULTS}

\section{Shear bond strength testing results:}

Shear Bond strength of randomly selected thirteen specimens of each group (the control and study groups) was determined by placing each specimen in a universal testing machine. Data was fed to the computer and analyzed using IBM SPSS software package version 20.0. (Armonk, NY: IBM Corp) The Kolmogorov-Smirnov test was used to verify the normality of distribution quantitative data was described using range (minimum and maximum), mean, standard deviation and median. Mann Whitney test was used for data analysis. Significance of the obtained results was judged at the 5\% level. The shear bond strength values are presented in (Table 1) and (Figure 2).
Table 1: Comparison of the shear bond strength between the Control group and Study group

\begin{tabular}{||l|c|c|c|c|}
\hline \hline & $\begin{array}{c}\text { Control group } \\
\text { Group I } \\
\text { Bonded to visiolign } \\
(\mathbf{n}=13)\end{array}$ & $\begin{array}{c}\text { Study group } \\
\text { Group II } \\
\text { Bonded to PMMA } \\
(\mathrm{n}=13)\end{array}$ & $\mathrm{U}$ & $\mathrm{p}$ \\
\hline Min.-Max. & $0.10-0.35$ & $0.41-0.59$ & & \\
Mean \pm SD. & $0.26 \pm 0.08$ & $0.50 \pm 0.07$ & $0.0^{*}$ & $<0.001^{*}$ \\
Median & 0.30 & 0.47 & & \\
\hline
\end{tabular}

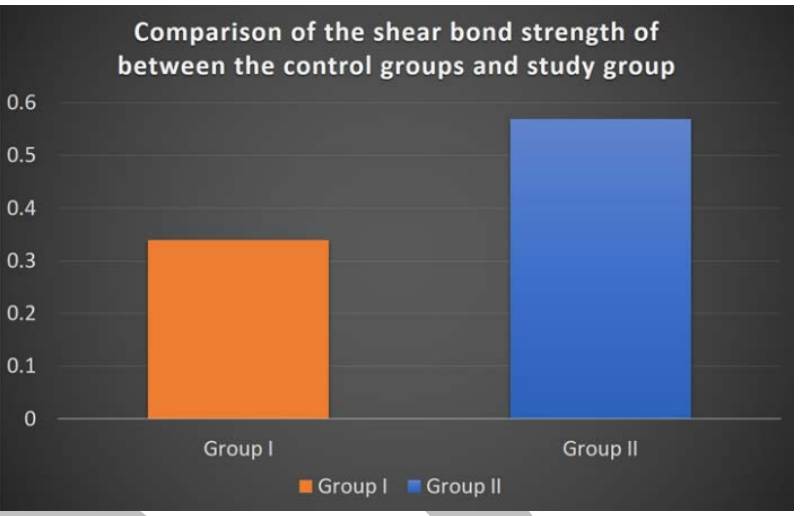

Figure 2: Comparison of the shear bond strength between the control group and study group

The mean value for shear bond strength was found to be higher for the study group (Group II) than control group (Group I). The statistical analysis revealed significant difference between the two groups where $(p \leq 0.05)$.

\section{Shear bond strength testing results after aging:}

Shear Bond strength of the other thirteen specimens of each group (the control and study groups) was determined, after thermocycling in distilled water for 1000 cycles between $5^{\circ} \mathrm{C}$ and $55^{\circ} \mathrm{C}$. The specimens were then placed in a universal testing machine. Data was fed to the computer and analyzed using IBM SPSS software package version 20.0. (Armonk, NY: IBM Corp) The Kolmogorov-Smirnov test was used to verify the normality of distribution quantitative data was described using range (minimum and maximum), mean, standard deviation and median. Mann Whitney test was used for data analysis. Significance of the obtained results was judged at the $5 \%$ level. The shear bond strength values are presented in (Table 2) and (Figure 3).

Table 2: Comparison between the shear bond strength of the control and study groups after aging

\begin{tabular}{|c|c|c|c|c|}
\hline \hline & $\begin{array}{c}\text { Control group } \\
\text { Group I } \\
\text { Bonded to visiolign } \\
(\mathrm{n}=13)\end{array}$ & $\begin{array}{c}\text { Study group } \\
\text { Group II } \\
\text { Bonded to PMMA } \\
(\mathrm{n}=13)\end{array}$ & $\mathrm{U}$ & $\mathrm{p}$ \\
\hline Min. - Max. & $0.10-0.35$ & $0.41-0.59$ & & \\
Mean \pm SD. & $0.26 \pm 0.08$ & $0.50 \pm 0.07$ & $0.0^{*}$ & $<0.001^{*}$ \\
Median & 0.30 & 0.47 & & \\
\hline
\end{tabular}




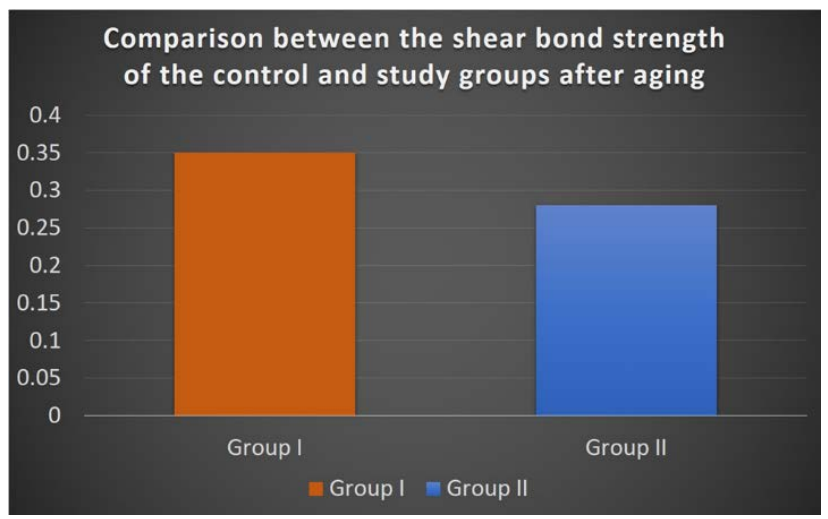

Figure 3: Comparison between the shear bond strength of the control and study groups after aging

The mean value of shear bond strength was found to be the same for the control and study groups. The statistical analysis revealed no significant difference between the two groups where $(\mathrm{p} \leq 0.05)$.

\section{Effect of Thermocycling:}

A comparison between the results of each study group before and after thermocycling were performed. Data was fed to the computer and analyzed using IBM SPSS software package version 20.0. (Armonk, NY: IBM Corp) The Kolmogorov-Smirnov test was used to verify the normality of distribution quantitative data was described using range (minimum and maximum), mean, standard deviation and median. Mann Whitney test was used for data analysis. Significance of the obtained results was judged at the $5 \%$ level. The shear bond strength values are presented in (Table 3) and (Figure 4).

Table 3: Comparison of the effect of aging for the control and study groups

\begin{tabular}{|l|c|c|c|c|}
\hline & $\begin{array}{c}\text { Before Aging } \\
(\mathbf{n}=\mathbf{1 3})\end{array}$ & $\begin{array}{c}\text { After Aging } \\
(\mathbf{n}=\mathbf{1 3})\end{array}$ & $\mathbf{U}$ & $\mathbf{p}$ \\
\hline Control group & & & & \\
Group I & & & & \\
Bonded to visiolign & & & & \\
$\quad$ Min. - Max. & $0.10-0.35$ & $0.10-0.36$ & & \\
$\quad$ Mean \pm SD. & $0.26 \pm 0.08$ & $0.27 \pm 0.08$ & 76.0 & 0.662 \\
$\quad$ Median & 0.30 & 0.30 & & \\
\hline \hline Study group & & & & \\
Group II & & & & \\
Bonded to PMMA & & $0.26-0.28$ & & \\
$\quad$ Min. - Max. & $0.41-0.59$ & $0.27 \pm 0.01$ & $0.0^{*}$ & $<0.001^{*}$ \\
$\quad$ Mean \pm SD. & $0.50 \pm 0.07$ & 0.27 & & \\
$\quad$ Median & 0.47 & &
\end{tabular}

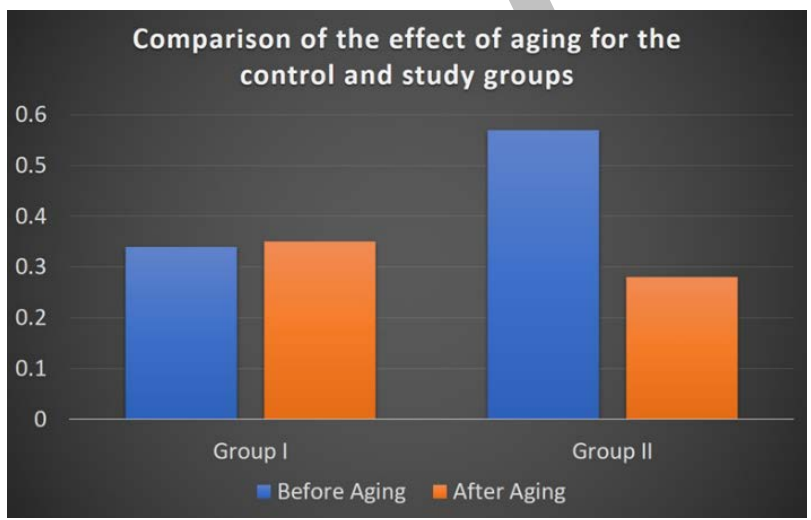

Figure 4: Comparison of the effect of aging for the control and study groups
For group I, the mean shear bond strength was found to be higher after aging. The statistical analysis revealed no significant difference where $(\mathrm{p} \leq 0.05)$, while in group II, the mean shear bond strength was found to be higher before aging. The statistical analysis revealed significant difference where $(\mathrm{p} \leq 0.05)$.

\section{DISCUSSION}

A new restoration material (poly-ether-ether-ketone [PEEK]) has been successfully used over the last few years in the medical field, and specially in orthopedics (22).

Although PEEK in general has been successfully used for years in orthopedics and medical technology, it has only recently been used in dentistry. Thus, studies evaluating the properties of this material are limited. The existing reports on this material are generally in vitro, emphasizing the need for further studies.

The grayish and opaque color of PEEK limits the application as a sole restoration. Therefore, PEEK needs to combine with esthetic resin material. However, the bond strength of the material is low when combined with resin materials because of the inert chemical performance, low surface energy, and surface modification resistance of PEEK. Thus, improving the surface properties of PEEK has become a research hotspot (21).

The choice of the tested adhesives was based on recommendations of the PEEK manufacturer. The user's manual suggests primarily the use of Visio-link to create a sufficient bond strength between the PEEK surface and different resins (23).

The normal low surface energy of polymers leads to adhesive problems, especially concerning the wetting and interaction between two polymers (24). The bonding improved simultaneously with the wetting, which meant that the surface energy increased (24). In this study, the increase in surface energy was achieved by air-abrasion performed before the bonding process.

Shear bond strength (SBS) test was employed because it can easily and rapidly reflect the clinical situation. SBS tests are more appropriate for evaluating adhesive capabilities of luting cements to composites. Any change in the surface treatment of the material may affect the shear strength, which is related to mechanical and chemical adhesion (25).

Studies showed that treatment using silica coating and using multifunctional acrylates containing primer resulted in durable bonding to PEEK surfaces (26). In the current study, no further pretreatment was performed after airborneparticle abrasion and adhesive treatments using Visio.link.

In another study the air-abraded (50 $\mu \mathrm{m}, 0.2 \mathrm{MPa})$ PEEK surfaces conditioned with adhesive systems Visiolink (40.0-69.0 MPa) or Signum PEEK Bond I + II (41.3$57.5 \mathrm{MPa}$ ) showed similar or higher TBS results compared to those obtained with other framework materials tested in previous studies (23).

Although the airborne-particle abrasion increases the surface area and allows a better infiltration of the adhesive material, the bonding is still predominantly characterized by mechanical interactions between the PEEK surface and the adhesive material. In contrast to this, the veneering material is additionally bonded by chemical means to the adhesive Visio.link layer and therefore creates a stronger bond (24).

It seems that MMA monomers are important contributors of increased bond strength between PEEK and veneering resins. This was also supported by the study of 
Kern \& Lehmann, which showed that a durable bonding to PEEK could only be achieved using the multifunctional methacrylate containing resin varnish (Luxatemp Glaze \& Bond) on air-abraded surfaces to create substantial chemical bonding to PEEK (26). That study also concluded that the use of phosphate monomer containing primer on air abraded PEEK did not result in any adhesion.

It is generally accepted that, for MMA/PMMA denture base materials, grinding the surface of the denture tooth and wetting it with monomer is a pre-requisite to obtain a clinically reliable bond strength (27). The bonding mechanism is believed to be based on an interpenetrating network or a covalent bond (27).

The results of this study showed that the initial shear bond strength of PMMA to PEEK was higher than that of composite resin to PEEK. The difference in shear bond strength values was statistically significant.

A previous study tested the bond strength of resin composite cements after conditioning using Visio-link and VP connect on air-abraded PMMA CAD/CAM materials (28). The conditioning liquids enhanced bond strength to PMMA within the used resin composite cements. MMA monomer in contact with industrially polymerized PMMA results in swelling of the polymers caused by the monomer diffusion process resulting in dissolving the PMMA surface and also increasing the TBS (29). This could explain the presence of higher SBS between the PMMA and the PEEK in the current study.

Furthermore, Previous investigations have stated that because of the process of post-polymerization of the adhesive system and the veneering composite resin, higher bond strength values could be observed with thermal changes (23). This also could be an explanation to the increased initial SBS of PMMA to PEEK due to the elevation of temperature during PMMA heat curing process.

Storage and thermocycling are the most often used artificial aging methods for simulating oral conditions in laboratory testing of bonding durability (26). This minimal requirement of simulating oral conditions seems necessary before clinical recommendations can be provided, even with caution. Artificial aging by the procedure of thermocycling may act in two different ways on the SBS; on one side it may lead to an increase of the bond strength, caused by the post-polymerization in the contact area of the PEEK surface, the adhesive and the veneering cement, while on the other side, the thermal stress may lead to mechanical stress of the bonding area caused by different volumetric changes of the concerned materials (23).

In the current study, after the aging process, the shear bond strength of PMMA to PEEK decreased while that of composite resin to PEEK remained unchanged. There was no significant difference between the shear bond strength of both PMMA and composite resin to PEEK.

In a study evaluating the strengthening of PMMA with preimpregnated fibers, it has been reported that the strengthening effect of preimpregnated fibers is more evident in auto- rather than in heat-polymerized acrylic resins (30). These authors suggested that the lower properties found with heat-polymerized acrylics could be due to decreased adhesive strength at the impregnating resin-PMMA interface, potentially caused by insufficient monomer wetting or differences in the thermal expansions between the reinforcement and the acrylic resin. These suggestions may be applicable to the results of the current study where the SBS of PMMA to PEEK decreased after thermocycling process.

One of the negative properties of PMMA is water absorption (31). This can be explained by the fact that a higher amount of resin matrix in combination with a lower content of filler particles results in higher water absorption (32). This phenomenon explains the decrease in SBS of PMMA to PEEK after thermocycling.

Up to date, there are no studies evaluating the bond between PEEK and PMMA, hence the importance of this study to evaluate the possibility of PEEK denture base relining with PMMA.

\section{CONCLUSION}

Within the limitation of an in vitro study, the following could be concluded:

1. PMMA can be used as a veneering material with PEEK with comparable results to traditionally used composite resin.

2. Owing to the adequate bond strength between PEEK and PMMA, PEEK frameworks could be relined in the traditional method similar to metal frameworks.

\section{CONFLICT OF INTEREST}

The authors declare that they have no conflicts of interest.

\section{REFERENCES}

1- Kurtz SM, Devine JN. PEEK biomaterials in trauma, orthopedic, and spinal implants. Biomaterials. 2007;28:4845-69.

2- Dawson P, Blundell D. X-ray data for poly (aryl ether ketones). Polymer. 1980;21:577-8.

3- Cebe P, Hong S-D. Crystallization behaviour of poly (etherether-ketone). Polymer. 1986;27:1183-92.

4- Jenkins M, Hay J, Terrill N. Structure evolution in melt crystallised PEEK. Polymer. 2003;44:6781-7.

5- Kumar S, Anderson DP, Adams WW. Crystallization and morphology of poly (aryl-ether-ether-ketone). Polymer. 1986;27:329-36.

6- Fratini A, Cross E, Whitaker R, Adams W. Refinement of the structure of PEEK fibre in an orthorhombic unit cell. Polymer. 1986;27:861-5.

7- Staniland P, Wilde C, Bottino F, Di Pasquale G, Pollicino A, Recca A. Synthesis, characterization and study of the thermal properties of new polyarylene ethers. Polymer. 1992;33:1976-81.

8- Liao K. Performance characterization and modeling of a composite hip prosthesis. Experimental Techniques. 1994;18:33-8.

9- Kelsey DJ, Springer GS, Goodman SB. Composite implant for bone replacement. Journal of composite materials. 1997;31:1593-632.

10-Rosentritt M, Preis V, Behr M, Sereno N, Kolbeck C. Shear bond strength between veneering composite and PEEK after different surface modifications. Clinical oral investigations. 2015;19:739-44.

11-Egusa H, Ko N, Shimazu T, Yatani H. Suspected association of an allergic reaction with titanium dental implants: a clinical report. The Journal of prosthetic dentistry. 2008;100:344-7.

12-Andreiotelli M, Wenz HJ, Kohal RJ. Are ceramic implants a viable alternative to titanium implants? A systematic literature review. Clinical Oral Implants Research. 2009;20:32-47. 
13-Prochazkova J, Podzimek S, Tomka M, Kucerova H, Mihaljevic M, Hana K, et al. Metal alloys in the oral cavity as a cause of oral discomfort in sensitive patients. Neuro endocrinology letters. 2006;27:53-8.

14-Steele J, Treasure E, O'sullivan I, Morris J, Murray J. Adult dental health survey 2009: Transformations in British oral health 1968-2009. British dental journal. 2012;213:523-7.

15-McCord JF, Grey NJ, Winstanley RB, Johnson A. A clinical overview of removable prostheses: Introduction. Dental update. 2002;29:375-81.

16-Behr M, Zeman F, Passauer T, Koller M, Hahnel S, Buergers R, et al. Clinical performance of cast claspretained removable partial dentures: a retrospective study. International Journal of Prosthodontics. 2012;25.

17-Fueki K, Ohkubo C, Yatabe M, Arakawa I, Arita M, Ino S, et al. Clinical application of removable partial dentures using thermoplastic resin-Part I: Definition and indication of non-metal clasp dentures. Journal of prosthodontic research. 2014;58:3-10.

18-Kistler F, Adler S, Kistler S. PEEK-Hochleistungskuns tstoffeimimplantat-prothetischen Workflow. Implantologie J. 2013;7:17-42.

19-Bilgin MS, Baytaroğlu EN, Erdem A, Dilber E. A review of computer-aided design/computer-aided manufacture techniques for removable denture fabrication. European journal of dentistry. 2016;10:286.

20-Piwowarczyk A, Lauer H-C, Sorensen JA. In vitro shear bond strength of cementing agents to fixed prosthodontic restorative materials. The Journal of prosthetic dentistry. 2004;92:265-73.

21-Noiset O, Schneider Y-J, Marchand-Brynaert J. Adhesion and growth of $\mathrm{CaCo} 2$ cells on surface-modified PEEK substrata. Journal of Biomaterials Science, Polymer Edition. 2000;11:767-86.

22-Zoidis P, Papathanasiou I, Polyzois G. The Use of a Modified Poly-Ether-Ether-Ketone (PEEK) as an Alternative Framework Material for Removable Dental Prostheses. A Clinical Report. Journal of Prosthodontics. 2016;25:580-4.

23-Stawarczyk B, Keul C, Beuer F, Roos M, Schmidlin PR. Tensile bond strength of veneering resins to PEEK: impact of different adhesives. Dental materials journal. 2013;32:441-8.

24-Taufall S, Eichberger M, Schmidlin PR, Stawarczyk B. Fracture load and failure types of different veneered polyetheretherketone fixed dental prostheses. Clinical oral investigations. 2016;20:2493-500.

25-Aboushelib MN. Evaluation of zirconia/resin bond strength and interface quality using a new technique. Journal of Adhesive Dentistry. 2011;13:255.

26-Kern M, Lehmann F. Influence of surface conditioning on bonding to polyetheretherketon (PEEK). Dental Materials. 2012;28:1280-3.
27-Chung R, Clark R, Darvell B. The bonding of cold-cured acrylic resin to acrylic denture teeth. Australian dental journal. 1995;40:241-5.

28-Baehr N, Keul C, Edelhoff D, Eichberger M, Roos M, Gernet W, et al. Effect of different adhesives combined with two resin composite cements on shear bond strength to polymeric CAD/CAM materials. Dental materials journal. 2013;32:492-501.

29-Vallittu P, Ruyter I. The swelling phenomenon of acrylic resin polymer teeth at the interface with denture base polymers. The Journal of prosthetic dentistry. 1997;78:1949.

30-Kanie T, Arikawa H, Fujii K, Ban S. Light-curing reinforcement for denture base resin using a glass fiber cloth pre-impregnated with various urethane oligomers. Dental materials journal. 2004;23:291-6.

31-Coy HD. Direct resin fillings. The Journal of the American Dental Association. 1953;47:532-7.

32-Musanje L, Darvell B. Aspects of water sorption from the air, water and artificial saliva in resin composite restorative materials. Dental Materials. 2003;19:414-22.

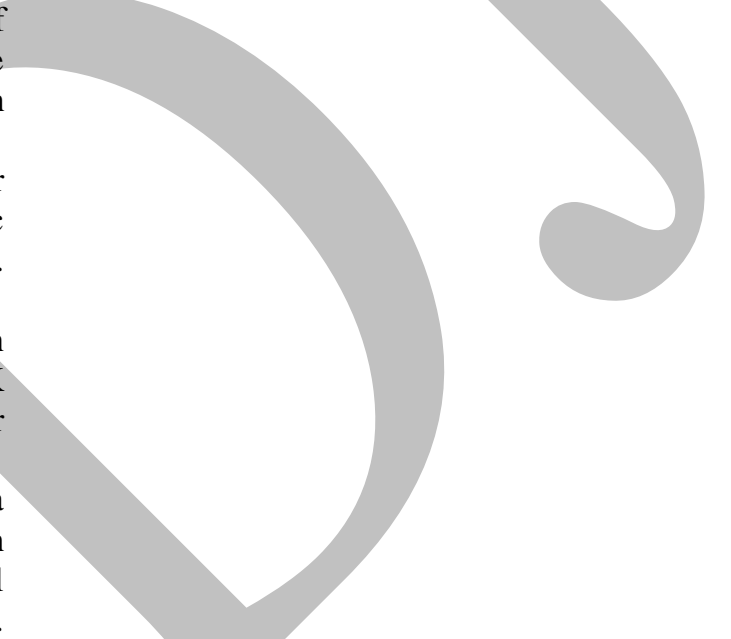

